\title{
Die Vergütung nichtärztlicher Leistungen Sozialpädiatrischer Zentren
}

\section{Einleitung}

Die Vergütung nichtärztlicher sozialpädiatrischer Leistungen Sozialpädiatrischer Zentren ist seit langem ein streitträchtiges Thema. In jüngster Zeit beschäftigt das Thema immer wieder die zuständigen Schiedsstellen, und im vergangenen Jahr wurde eine längere Auseinandersetzung durch ein Urteil des LSG Berlin-Brandenburg zum Abschluss gebracht ${ }^{1}$.

Sozialpädiatrie versteht sich als ,eine Querschnittswissenschaft der Kinderheilkunde und Jugendmedizin, die sich mit den Beziehungen zwischen Gesundheit, Krankheit und Entwicklung der Kinder einerseits und den äußeren Lebensbedingungen der Kinder andererseits befasst.“2. In individualmedizinischer Hinsicht bemüht sich Sozialpädiatrie um „Kinder und Jugendliche 'mit besonderen Bedürfnissen' (behinderte, chronisch kranke sowie mißhandelte, mißbrauchte und vernachlässigte Kinder)“. Sozialpädiatrie sieht für den Erfolg präventiver, therapeutischer und rehabilitativer Interventionen „die Einflussnahme auf das soziale Umfeld“ als entscheidend $\mathrm{an}^{3}$. Sozialpädiatrische Versorgung erfolgt typischerweise in multiprofessionellen Teams ärztlicher und nichtärztlicher Akteure - interdisziplinär, aber stets unter ärztlicher Verantwortung; die nichtärztlichen Akteure solcher Teams verfügen teils über medizinische Qualifikationen (z.B. Physiotherapeuten), teils sind sie pädagogisch ausgebildet (Heiloder Sozialpädagogen). Überwiegend wird die sozialpädiatrische Versorgung ambulant durchgeführt, zunächst - in der Grundversorgung - im Rahmen der vertragsärztlichen Versorgung durch Fachärzte für Kinder und Jugendmedizin und auch durch Fachärzte für Kinderund Jugendpsychiatrie, dann - auf gehobener Versorgungsstufe, aber gleichwohl meist ambulant - auf der Grundlage fachärztlicher Überweisungen durch Sozialpädiatrische Zentren, von denen es in Deutschland etwa 150 gibt $^{4}$.

Die Ausrichtung auch auf das soziale Umfeld und die Beteiligung medizinferner Berufsgruppen dürfte ein Grund dafür sein, dass die Einordnung sozialpädiatrischer Interventionen in das Recht der gesetzlichen Krankenversicherung Schwierigkeiten bereitet. Dies ist deutlich geworden, als vor 30 Jahren Regelungen zur sozialpädiatrischen Versorgung in das Gesetzesrecht der gesetzlichen Krankenversicherung aufgenommen wurden. Spätere Modifikationen der einschlägigen Vorschriften haben die Rechtslage immer komplizierter und undurchsichtiger gemacht, wohl auch deshalb, weil die Belange einer ganzen Reihe von Interessengruppen berührt sind.

\footnotetext{
${ }^{1}$ LSG Berlin- Brandenburg, Urt. v. 12.10.2018 - L 24 KA 37/17 KL, abgedruckt in diesem Heft auf den S. .....; vgl. auch den Beschluss des LSG aus dem Eilverfahren: Beschl. v. 27.6.2017 - L 24 KA 35/17 KL ER, KRS 2017, S. $410-416$.

${ }^{2}$ Schlack, Sozialpädiatrie - Definition und Aufgaben, in: Schlack/Landolt/Sennhauser, Sozialpädiatrie und Recht, in: Lenze/Schaub/Schulte/Spranger (Hrsg.), Pädiatrie, 3. Aufl., 2007, S. 105 - 132; vgl. auch Schlack, Stichwort „Sozialpädiatrie“, in: Deutscher Verein für öffentliche und private Fürsorge (Hrsg.), Fachlexikon der sozialen Arbeit, 9. Aufl., 2017, S. 835 f.

${ }^{3}$ Schlack, Stichwort „Sozialpädiatrie“, in: Deutscher Verein für öffentliche und private Fürsorge (Hrsg.), Fachlexikon der sozialen Arbeit, 9. Aufl., 2017, S. 835.

${ }^{4}$ Schlack, Stichwort „Sozialpädiatrische Zentren“, in: Deutscher Verein für öffentliche und private Fürsorge (Hrsg.), Fachlexikon der sozialen Arbeit, 9. Aufl., 2017, S. 836; Schlack spricht von 130 Sozialpädiatrischen Zentren; die Deutsche Gesellschaft für Sozialpädiatrie und Jugendmedizin e.V. verzeichnet auf ihrer Website derzeit 150 Zentren, vgl. www.dgspj.de/category/sozialpaedagogische-zentren/, abgerufen am 15.5.2019.
} 
Dieser Beitrag wird zunächst die aktuelle Gesetzeslage darstellen und ihre Vorgeschichte nachzeichnen. Vor diesem Hintergrund kann dann die vom LSG Berlin-Brandenburg entschiedene Problematik vorgestellt und die Lösung des LSG bewertet werden.

\section{Die Entwicklung der Regelungen über die sozialpädiatrische Versorgung}

\section{Gegenwärtige Gesetzeslage}

Zur sozialpädiatrischen Versorgung findet sich heute im SGB V zunächst mit § 43a eine leistungsrechtliche Vorschrift. Danach haben versicherte Kinder Anspruch auf nichtärztliche sozialpädiatrische Leistungen, wenn diese unter ärztlicher Verantwortung erbracht werden und erforderlich sind, um eine Krankheit zum frühestmöglichen Zeitpunkt zu erkennen und einen Behandlungsplan aufzustellen (Abs. 1 Hs. 1). Als Beispiele für nichtärztliche sozialpädiatrische Leistungen werden psychologische, heilpädagogische und psychosoziale Leistungen benannt. Außerdem ist vorgesehen, dass versicherte Kinder Anspruch auf nichtärztliche sozialpädiatrische Leistungen haben, die unter ärztlicher Verantwortung in der ambulanten psychiatrischen Behandlung erbracht werden (Abs. 2).

Sozialpädiatrische Zentren können unter den in § 119 Abs. 1 SGB V genannten Voraussetzungen zur ambulanten sozialpädiatrischen Behandlung von Kindern ermächtigt werden; sie können eine Ermächtigung beanspruchen, wenn dies nötig ist, um eine ausreichende sozialpädiatrische Behandlung sicherzustellen (Abs. 1). Die Behandlung durch sozialpädiatrische Zentren muss auf die Kinder ausgerichtet werden, die wegen Art, Schwere oder Dauer ihrer Krankheit oder einer drohenden Krankheit nicht von geeigneten Ärzten oder in geeigneten Frühförderstellen behandelt werden können; mit den Ärzten und Frühförderstellen sollen die Zentren eng zusammenarbeiten (Abs. 2). Die Leistungen der sozialpädiatrischen Zentren werden nicht aus der Gesamtvergütung finanziert, sondern unmittelbar von den Krankenkassen vergütet (§ 120 Abs. 2 S. 1 SGB V). Die Vergütung wird von den Landesverbänden der Krankenkassen und den Ersatzkassen mit den Zentren vereinbart ( $\$ 120$ Abs. 2 S. 2); sie muss deren Leistungsfähigkeit bei wirtschaftlicher Betriebsführung gewährleisten ( $§ 120$ Abs. 2 S. 3). Es können Vergütungspauschalen vereinbart werden (§ 120 Abs. 3 S. 1). Kommt eine Vergütungsvereinbarung nicht zustande, wird die Vergütung durch die Schiedsstelle nach dem KHG festgesetzt (§ 120 Abs. 4 S. 1 mit $§ 18 \mathrm{a}$ Abs. 1 KHG).

\section{Einführung erster Regeln über sozialpädiatrische Zentren durch das GRG (1988) und beginnender Streit}

Die genannten Regelungen über die Ermächtigung sozialpädiatrischer Zentren und über die Vergütung der dort erbrachten Leistungen gehen zurück auf das GRG aus dem Jahr $1988^{5}$. Sie gelten weithin unverändert seit dem Inkrafttreten des SGB V am 1.1.1989 - mit einer Ausnahme: Der ursprüngliche $\S 119$ SGB V war in Bezug auf einen wesentlichen Punkt anders ausgestaltet als heute: Er enthielt als Absatz 2 eine Regel über den Inhalt der sozialpädiatrischen Behandlung; diese, so hieß es damals, „umfaßt die ärztlichen und nichtärztlichen Leistungen, insbesondere auch psychologische Leistungen sowie ergänzende Leistungen zur Rehabilitation ( $§ 43$ [a.F.]), die erforderlich sind, um insbesondere auch mit der in $\S 11$ Abs. 2

\footnotetext{
${ }^{5}$ Gesetz zur Strukturreform im Gesundheitswesen (Gesundheits-Reformgesetz - GRG) v. 20.12.1988, BGB1. I S. 2477; vgl. dazu die Begründung zum Gesetzentwurf der Fraktionen der CDU/CSU und der FDP v. 3.5.1988, BT-Drs. 11/2237, S. 202 f.
} 
[a.F.] genannten Zielsetzung eine Krankheit zum frühestmöglichen Zeitpunkt zu erkennen, zu verhindern, zu heilen oder in ihren Auswirkungen zu mildern“.

Diese Regelung führte alsbald zum Streit. Die Sozialpädiatrischen Zentren waren angesichts der weit gefassten Regel des $\S 119$ Abs. 2 a.F. der Ansicht, alle bei ihnen erbrachten ärztlichen und nichtärztlichen Leistungen seien als Gesamtleistung von den Krankenkassen zu vergüten ${ }^{6}$. Die Kassen hingegen vergüteten nur diejenigen Teilleistungen der Zentren, die auch in den leistungsrechtlichen Vorschriften vorgesehen waren. Bei den Vergütungsvereinbarungen waren aus Sicht der Krankenkassen wegen des Anspruchs der Versicherten auf ärztliche Behandlung nach $\S 27$ Abs. 1 S. 1 und S. 2 Nr. 1 SGB V selbstverständlich die ärztlichen Leistungen zu berücksichtigen, außerdem ergänzende Leistungen zur Rehabilitation auf Basis der $\S \S 27$ Abs. 1 S. 1 und S. 2 Nr. 6, 43 (a.F.) SGB V, zu denen auch gewisse nichtärztliche Leistungen zu rechnen seien, wie z.B. Krankengymnastik, Ergotherapie oder Logopädie, nicht jedoch pädagogische, soziale und psychosoziale Leistungen; für erforderliche Leistungen der interdisziplinären Abstimmung der diagnostischen und therapeutischen Maßnahmen sah sich die Kassenseite nur teilweise für verpflichtet $a^{7}$. Vor diesem Hintergrund verfuhr man in der Praxis nach Vereinbarungen, die die Übernahme des Teils der Kosten, für den sich die Kassen nicht zuständig fühlten, durch die Sozialhilfeträger vorsahen - in der Erwartung einer gerichtlichen Klärung der Streitfrage, zu der es aber nicht kam ${ }^{8}$.

\section{Klärungsversuch durch Einführung des § 43a SGB V (a.F.) im Jahr 1991}

Es war der Gesetzgeber, der 1991 einen Klärungsversuch unternahm. Mit § 43a (a.F.) SGB V wurde eine leistungsrechtliche Regelung in Bezug auf nichtärztliche sozialpädiatrische Leistungen eingeführt und zugleich $\S 119$ Abs. 2 a.F. gestrichen ${ }^{9}$. Die neue Regelung gilt noch heute, als $\S 43 \mathrm{a}$ Abs. 1 Hs. 1. Sie gewährt versicherten Kindern einen Anspruch auf nichtärztliche sozialpädiatrische Leistungen. Anders als im entfallenen $\S 119$ Abs. 2 a.F. ist hier allerdings nicht mehr uneingeschränkt die Rede von ärztlichen und nichtärztlichen Leistungen; es sollen vielmehr nichtärztliche sozialpädiatrische Leistungen erbracht werden, ,wenn sie erforderlich sind, um eine Krankheit zu erkennen und einen Behandlungsplan aufzustellen“; die Phase der Durchführung therapeutischer Maßnahmen ist in dieser Formulierung ausgespart.

Die Einführung des $\S 43 a$ a.F. führte nicht zu einer Klärung des Streits um die Reichweite der Einstandspflichten der Krankenkassen ${ }^{10}$. Deshalb wurden im Verlauf des Jahres 1992 zwei Gesprächsrunden beim Bundesministerium für Gesundheit durchgeführt, an denen Vertreter der Spitzenverbände der beteiligten Akteure teilnahmen. Bei diesen Runden wurden informelle Absprachen zu einem Kostenteilungsmodell getroffen, das dann für die nächsten 25 Jahre die Vergütungspraxis prägen sollte. Man verständigte sich auf eine Teilung der Kosten zwischen Krankenkassen und Sozialhilfeträgern im Verhältnis 85 : 15. Dabei ging man von

\footnotetext{
${ }^{6}$ Schulin, Krankenversicherungsrechtliche Beurteilung von Leistungen in Sozialpädiatrischen Zentren (Rechtsgutachten für die Deutsche Gesellschaft für Sozialpädiatrie e.V.), 1995, S. 38.

${ }^{7}$ Nrn. 4 und 7 der Gemeinsamen Empfehlung der Spitzenverbände v. 16.10.1989, abgedruckt bei Schulin, Krankenversicherungsrechtliche Beurteilung von Leistungen in Sozialpädiatrischen Zentren, 1995, S. 37 f.

${ }^{8}$ Schulin, aaO (Fn. Xxx), S. 38.

${ }^{9}$ Art. 1 Nrn. 12 und 28 des Zweiten Gesetzes zur Änderung des Fünften Buchs Sozialgesetzbuch v. 20.12.1991, BGBl. I, S. 2325; dazu BT-Drs. 12/1154 (Regierungsentwurf), BT-Drs. 12/1387 und 12/1392 (Ausschuss); der vorherige $\S 119$ Abs. 3 wurde so zu $§ 119$ Abs. 2.

${ }^{10}$ Hierzu Schulin, aaO (Fn xxx); ferner Breimeier, Sozialpädiatrische Zentren. Rechtsunsicherheit hinsichtlich der Finanzierung dauert an, KH 1992, S. 538 (541 f.), Abdruck einer Gesprächsnotiz ebd. S. 542 f.; vgl. auch die Darstellung im ausführlichen Schiedsspruch der Schiedsstelle nach § 18a KHG für das Land Brandenburg v. 9.5.2017, KrV 2017, S. 212 ff. (214, 1.Sp.).
} 
einer Einstandspflicht der Krankenkassen für die Kosten der Ärzte und des nichtärztlichen Personals mit medizinischer Ausbildung aus; die Kosten des nichtärztlichen Personals mit pädagogischer Ausbildung und mit Abschlüssen als Sozialarbeiter oder Sozialpädagoge wurden den Sozialhilfeträgern zugewiesen.

\section{Komplikation: Regelungen des SGB IX über Leistungen der Früherkennung und Frühförderung (2001)}

Mit dem SGB IX wurden im Jahr 2001 Vorschriften über Leistungen der Früherkennung und Frühförderung eingeführt, die sich auf ärztliche und nichtärztliche sozialpädiatrische Leistungen beziehen und überdies auch auf nichtärztliche heilpädagogische Leistungen, die außerhalb ärztlicher Verantwortung erbracht werden. Diese Regelungen haben die Intransparenz der Gesetzeslage weiter verstärkt. Jüngst hat dann das $\mathrm{BTHG}^{11}$ diese Regelungen mit neuen Paragraphennummern ausgestattet.

Früherkennung und Frühförderung für behinderte oder von Behinderung bedrohte Kinder gehören zu den vom zuständigen Rehabilitationsträger zu erbringenden Leistungen der medizinischen Rehabilitation (§ 26 Abs. 2 Nr. 1 SGB IX a. F. / § 42 Abs. 2 Nr. 2 SGB IX n.F.). Dazu zählen auch nichtärztliche sozialpädiatrische (psychologische, heilpädagogische und psychosoziale) Leistungen, die der Frühdiagnostik und der Aufstellung eines Behandlungsplans dienen, wenn diese Leistungen unter ärztlicher Verantwortung erbracht werden ( $\$ 30$ Abs. 1 S. 1 Nr. 2 SGB IX a.F. / § 46 Abs. 1 Nr. 2 SGB IX n.F.). Diese Leistungen werden zusammen mit heilpädagogisch-therapeutischen Fördermaßnahmen außerhalb ärztlicher Verantwortung als sog. Komplexleistungen erbracht ( $\S 30$ Abs. 1 S. 2, 56 SGB IX a.F. / $\S 46$ Abs. 3 S. 1, 79 SGB IX n.F). Die beteiligten Rehabilitationsträger sollen sich über die Übernahme oder Aufteilung der Kosten einigen (§30 Abs. 3 S. 1 SGB IX a.F. / vgl. auch $\S 46$ Abs. 5 SGB IX n.F.). Zugleich wurde in $\S 43$ a SGB V ein Halbsatz eingefügt, nach dem $\S 30$ SGB IX a.F. (§ 46 SGB IX n.F.) unberührt bleibt. Ergänzende Regelungen enthält die auf der Grundlage einer Verordnungsermächtigung im SGB IX (§ 32 Nr. 1 a.F. / § 48 Nr. 1 n.F.) im Jahr 2003 erlassene Frühförderungsverordnung (FrühV) ${ }^{12}$. Danach werden Maßnahmen der Früherkennung und Frühförderung in erster Linie von familien- und wohnortnahen Interdisziplinären Frühförderstellen ( 33 FrühV), aber auch von Sozialpädiatrischen Zentren ( $\$ 4$ FrühV) erbracht. Sozialpädiatrische Zentren, die als Frühförderstellen in diesem Sinne tätig werden und Leistungen auch mit einem Sozialhilfeträger bzw. einem Träger der Eingliederungshilfe abrechnen wollen, müssen mit dem Träger zuvor eine Leistungs- und Vergütungsvereinbarung abschließen $(\S 125 \text { SGB IX })^{13}$.

\footnotetext{
${ }^{11}$ Art. 1 des Gesetzes zur Stärkung der Teilhabe und Selbstbestimmung von Menschen mit Behinderungen (Bundesteilhabegesetz) v. 23.12.2016, BGB1 I, S. 3234,

12 Verordnung zur Früherkennung und Frühförderung behinderter und von Behinderung bedrohter Kinder (Frühförderungsverordnung - FrühV) v. 24.6.2003, BGB1. I, S. 998), heute in der Fassung des Art. 23 des Gesetzes v. 23.12.2016, BGB1. I S. 3234.

${ }^{13}$ Vgl. auch - bezogen auf Thüringen - den Hinweis noch auf $\S 75$ Abs. 3 SGB XII in $\S 5$ Abs. 6 der Rahmenvereinbarung für den Freistaat Thüringen vom 1.5.2010 zur Umsetzung der Verordnung zur Förderung von Früherkennung und Frühförderung behinderter und von Behinderung bedrohter Kinder (Frühförderungsverordnung - FrühV) vom 24.6.2003.
} 


\section{Weitere Komplikation: Einführung eines Anspruchs auf nichtärztliche sozialpädiatri- sche Leistungen bei ambulanter psychiatrischer Behandlung im Jahr 2009 (§ 43a Abs. 2 SGB V)}

In Bezug auf einen Ausschnitt der sozialpädiatrischen Versorgung durch Vertragsärzte haben die Krankenkassen bemerkenswerterweise bereits seit den 90er Jahren auch nichtärztliche sozialpädiatrische Leistungen jenseits der Phase der Diagnostik finanziert. Diese Finanzierungspraxis bezog sich auf sozialpädiatrische Behandlung durch Fachärzte für Kinder- und Jugendpsychiatrie, wenn diese auch Praxismitarbeiter ohne medizinische Ausbildung, insbesondere Heilpädagogen und Sozialarbeiter einsetzten. Hintergrund dieser Praxis war eine Klausel in der Regelung des SGB V über die Gesamtvergütung: 1988 waren in Bezug auf sozialpädiatrische Behandlung nicht allein die oben dargestellten Regelungen zu den Sozialpädiatrischen Zentren und zur Vergütung von deren Leistungen durch die Krankenkassen in das Gesetz aufgenommen worden, sondern auch eine Vorschrift über die Vergütung nichtärztlicher sozialpädiatrischer Leistungen, die durch Vertragsärzte erbracht werden. Die damals als $\S 85$ Abs. 2 S. 3 SGB V (a.F.) eingeführte Regelung lautete: „Die Vertragsparteien (sc.: des Gesamtvertrages) sollen auch eine angemessene Vergütung für nichtärztliche Leistungen im Rahmen sozialpädiatrischer und psychiatrischer Tätigkeit vereinbaren“"14. Zum 1.1.1993 wurde diese Vorschrift inhaltlich unverändert in Satz 4 des $§ 85$ Abs. 2 SGB V verschoben ${ }^{15}$.

Zur Umsetzung dieser Regelung war erstmals 1994 eine bundesweite sog. SozialpsychiatrieVereinbarung zwischen den Parteien des Bundesmantelvertrages für die Ersatzkassen geschlossen worden ${ }^{16}$. Diese Vereinbarung ermöglichte als Vertragsärzten zugelassenen Fachärzten für Kinder- und Jugendpsychiatrie, Heilpädagogen oder Sozialpädagogen in ihre Praxisteams aufzunehmen und auch für deren Tätigkeit im Rahmen sozialpsychiatrischer Behandlung gegenüber der Kassenärztlichen Vereinigung Gebührenpauschalen abzurechnen. Bei den anderen Kassenarten gab es zum Teil vergleichbare Vereinbarungen auf Landesebene $^{17}$.

In den Jahren vor 2009 bemühten sich manche Krankenkassen darum, aus diesem Finanzierungsmodell auszusteigen, indem sie die einschlägigen gesamtvertraglichen Regelungen kündigten - die Existenz solcher Vereinbarungen war damals noch nicht zwingend vorgeschrieben. Der Gesetzgeber sah in diesem Rückzug der Krankenkassen eine Gefährdung der „etablierten Strukturen in der sozialpsychiatrischen ambulanten nichtärztlichen Versorgung von Kindern und Jugendlichen“ und schrieb, um „Einbrüche in der sozialpsychiatrischen Versorgung“ zu vermeiden, ${ }^{18}$ den Parteien der Gesamtverträge deshalb nun von Gesetzes wegen zwingend vor, die Vergütung nichtärztlicher Leistungen im Rahmen sozialpädiatrischer Tätigkeit bei Vertragsärzten gesamtvertraglich zu regeln; ergänzend wurde vorgegeben, dass das Nähere hierzu im Bundesmantelvertrag vereinbart werden müsse (§ 85 Abs. 2 S. 4 Hs. 1 bzw.

\footnotetext{
${ }^{14} \S 85$ Abs. 2 S. 3 SGB V i.d.F. des Gesetzes zur Strukturreform im Gesundheitswesen (Gesundheits-Reformgesetz - GRG) v. 20.12.1988, BGB1. I S. 2477.

${ }^{15}$ Aufgrund von Art. 1 Nr. 43 des Gesundheitsstrukturgesetzes v. 21.12.1992, BGBl I, S. 2266.

${ }^{16}$ Deutsches Ärzteblatt 1994, Heft 28-29, C-1267; dazu Schydlo, Die „Sozialpsychiatrie-Vereinbarung“, Deutsches Ärzteblatt 1994, Heft 30, S. A- $2057-2059$.

${ }^{17}$ Vgl. auch § 9 Abs. 2 der derzeit geltenden „Vereinbarung gemäß $\S 85$ Abs. 2 Satz 4 und $\S 43$ a SGB V über besondere Maßnahmen zur Verbesserung der sozialpsychiatrischen Versorgung von Kindern und Jugendlichen (Sozialpsychiatrie-Vereinbarung)“"(Anlage 11 zum BMV-̈̈, Stand 1.1.2019; die Vereinbarung mit Stand 1.7.2009 ist abgedruckt in DÄ 2009, Heft 31-32, Ausgabe A, S. 1578 ff.

${ }^{18}$ Zitate aus dem Regierungsentwurf zum Gesetz zur Änderung arzneimittelrechtlicher und anderer Vorschriften v. 16.3.2009, BT-Drs. 16/12256, S. 35.
} 
Hs. 2 SGB V n.F. ${ }^{19}$ ). Als leistungsrechtliches Pendant zu dieser Regelung wurde überdies die ebenfalls noch heute geltende Regelung des $\S 43 \mathrm{a}$ Abs. 2 SGB V ${ }^{20}$ eingeführt, derzufolge versicherten Kindern nichtärztliche sozialpädiatrische Leistungen zustehen, die unter ärztlicher Verantwortung in der ambulanten psychiatrischen Behandlung erbracht werden, und zwar ohne Beschränkung auf diagnostische Leistungen und Behandlungsplanung ${ }^{21}$. Dementsprechend enthält der Leistungskatalog der heute für alle Kassenarten geltenden SozialpsychiatrieVereinbarung 22 nach wie vor auch heilpädagogische und sozialtherapeutische Maßnahmen (Anlage 1 II Nr. 6).

\section{III. „Umfassende“ Leistungspflicht der Krankenkassen bei nichtärztlichen sozialpädiat- rischen Leistungen der Sozialpädiatrischen Zentren?}

\section{Streit um das Ende der Kostenteilung vor dem LSG Berlin Brandenburg: der Fall}

Zwischen den Krankenkassen und den Sozialpädiatrischen Zentren in Brandenburg waren seit 1998 Vergütungsvereinbarungen über Fallpauschalen geschlossen worden, von denen die Krankenkassen einen Anteil von $85 \%$ zu tragen hatten, während für die verbleibenden $15 \%$ eine Einstandspflicht der Sozialhilfeträger angenommen wurde. Dementsprechend war zuletzt für das Jahr 2014 folgende Klausel vereinbart worden: „Die Vertragspartner vereinbaren (...) eine Fallpauschale in Höhe von 339,83 Euro. Die zu Lasten der Krankenkassen abrechnungsfähige Fallpauschale [sc. je Patient + pro Quartal] beträgt (...) 288,85 Euro (85 v.H.). Der von Seiten der Krankenversicherung nicht gedeckte Betrag ist von den Sozialhilfeträgern aufzubringen." Eines der Sozialpädiatrischen Zentren in Brandenburg wollte diese Praxis für die Jahre 2015 und 2016 ändern und verlangte von den Kassen die Vereinbarung einer erhöhten Fallpauschale, die überdies von den Krankenkassen in vollem Umfang (100\%) übernommen werden sollte. Hintergrund dieser Forderung war offenbar die Weigerung einiger Sozialhilfeträger, einen Kostenanteil zu tragen. Die Kassenseite verweigerte jedoch eine ungekürzte Übernahme der pauschalierten Kosten (348,43 Euro), sondern bot lediglich eine Erhöhung der von ihr bisher getragenen Pauschale von 288,85 Euro auf 296,16 Euro an (=85 \% von 348,43 Euro). Das Zentrum rief die Schiedsstelle an, die am 16. Juni 2016 eine Fallpauschale in Höhe von 348,43 Euro beschloss: das Zentrum könne nicht darauf verwiesen werden, für einen Teil der sozialpädiatrischen Gesamtbehandlung die Sozialhilfeträger in Anspruch nehmen zu müssen. Gegen den Beschluss der Schiedsstelle klagte die AOK Nordost. Auf Antrag des Sozialpädiatrischen Zentrums ordnete das LSG zunächst im Juni 2017 die sofortige Vollziehung des Schiedsspruchs $a^{23}$. Im Oktober 2018 wies das LSG die Klage als unbegründet $\mathrm{ab}^{24}$. Die Revision wurde nicht zugelassen. Das Urteil ist rechtskräftig geworden.

Dem im Urteil des LSG wiedergegebenen Vortrag der Verfahrensbeteiligten lässt sich nicht im Detail entnehmen, wie die Fallpauschalen kalkuliert wurden. Da man sich aber auch in Brandenburg seit 1998 an dem oben dargestellten „Gesprächsrundenergebnis“ und der damals gefundenen „85/15-Regel“" orientiert hat, ist davon auszugehen, dass in die Kalkulation der

\footnotetext{
${ }^{19}$ In der Fassung des Art. 15 Nr. 5 des Gesetzes zur Änderung arzneimittelrechtlicher und anderer Vorschriften v. 17.7.2009, BGB1. I, S. 1990, in Kraft getreten am 23.7.2009.

${ }^{20}$ Art. 15 Nr. 04 des Gesetzes zur Änderung arzneimittelrechtlicher und anderer Vorschriften v. 17.7.2009, BGB1. I, S. 1990, in Kraft getreten am 23.7.2009; dazu BT-Drs. 16/13428, S. 75, 80 und S. 90.

${ }^{21}$ So ausdrücklich die Beschlussempfehlung des Ausschusses für Gesundheit BT-Drs. 16/13428, S. 80 (Begründung zu Änderungsantrag Nr. 11).

${ }^{22}$ Nachweis oben in Fn. Xxx.

${ }^{23}$ LSG Berlin-Brandenburg, Beschl. v. 27.6.2017 - L 24 KA 35/17 KL ER, KRS 2017, S. 410 - 416

${ }^{24}$ In diesem Heft ......
} 
ungekürzten Fallpauschale ein Anteil von $15 \%$ eingegangen ist, der die Kosten des Zentrums für sein nichtärztliches Personal mit pädagogischer Ausbildung und mit Abschlüssen als Sozialarbeiter oder Sozialpädagogen berücksichtigt. Vor diesem Hintergrund ist als zentrales Rechtsproblem der Auseinandersetzung die Frage anzusehen, ob die Krankenkassen auch Maßnahmen dieses Personals finanzieren müssen. Die Krankenkassen haben diese Frage verneint. Die Zentren, die Schiedsstelle und auch das LSG haben die Frage nach der umfassenden Finanzierungspflicht der Kassen bejaht; das LSG bezeichnet dieses Lösungskonzept als „Umfassungsgrundsatz“.

Bei der Beantwortung dieser Frage ist zunächst davon auszugehen, dass eine Finanzierung des genannten Teils des nichtärztlichen Personals des Zentrums durch die Kassen nur in Betracht kommt, wenn die von den besagten Mitarbeitern durchgeführten Maßnahmen von den betroffenen Kindern und Jugendlichen auch als Leistungen der gesetzlichen Krankenversicherung beansprucht werden können. Dieser Ausgangspunkt kommt zwar im Urteil des LSG nicht sehr klar zum Ausdruck. Er ist jedoch dem Gesetz deutlich zu entnehmen: Mit den Fallpauschalen werden ,die Leistungen .... der sozialpädiatrischen Zentren .... vergütet“ $(\S 120$ Abs. 2 S. 1 SGB V), und mit „Leistungen“ ist die ,ambulante (..) sozialpädiatrische (..) Behandlung“ (§ 119 Abs. 1 S. 1 SGB V), gemeint, die versicherte Kinder nach dem Leistungsrecht des SGB V beanspruchen können, also nach den $\S \S 27$ ff. des Gesetzes.

\section{Plausibilität der ,kleinen Lösung“ vor 2009}

Bei der Darstellung der Gesetzesgeschichte ist deutlich geworden, dass die Reichweite der Leistungspflicht der Sozialpädiatrischen Zentren von Anfang an nicht wirklich klar geregelt war. Nach der ursprünglichen Fassung des $\S 119$ SGB V sprach der Wortlaut des Gesetzes (Abs. 2 a.F.) freilich recht deutlich dafür, dass auch nichtärztliche Leistungen unterschiedlicher in den Zentren tätiger Berufsgruppen in die Leistungs- und Finanzierungspflicht einbezogen sein sollten; allerdings konnte man eine solche Auslegung des Gesetzes mit dem Argument anzweifeln, dass es sich bei § 119 SGB V „nur“ um eine Regelung aus dem Leistungserbringungsrecht handelt, der es an einer expliziten Entsprechung im Leistungsrecht fehlte. Die Einführung des $\S 43 \mathrm{a}$ SGB V a.F. (= $\S 43 \mathrm{a}$ Abs. 1 S. 1 SGB V) verankerte zwar die nichtärztlichen sozialpädiatrischen Leistungen ausdrücklich im Leistungsrecht, allerdings in einer Weise, die eher Argumente für die restriktive Lösung lieferte ${ }^{25}$, denn nichtärztliche Leistungen gehören danach zum Leistungsprogramm, soweit sie für die Diagnose und für die Erstellung des Behandlungsplans erforderlich sind; die eigentlichen therapeutischen Maßnahmen der dort genannten nichtärztlichen Akteure werden vom Wortlaut der Regelung aber gerade nicht einbezogen.

Schulin hat hierzu die Auffassung vertreten, § 43a SGB V a.F. könne nicht so verstanden werden, dass eine Leistungspflicht der Kassen in Bezug auf therapeutische Maßnahmen nichtärztlicher Akteure ausgeschlossen sein solle ${ }^{26}$. Eine solche Leistungspflicht ergebe sich vielmehr bereits aus den $\S \S 27$ Abs. 1 S. 1 u. 2 Nr. 1 mit 28 Abs. 1 SGB V, denn danach zählt zur ärztlichen Behandlung einer Krankheit ,,auch die Hilfeleistung anderer Personen, die von dem

\footnotetext{
${ }^{25}$ So Noftz in Hauck (Hrsg.), SGB V, § 43a Rn. 5 (Loseblatt, Stand 10. EL, XI 1992).

${ }^{26}$ Schulin, aaO. (xxxx), S. 57f., 71 und 72; ganz in diesem Sinne auch der Bundestagsabgeordnete Jagoda (CDU/CSU): „Für die Therapiephase gilt dann das Krankenkassenrecht“, bzw.: er habe stets gesagt, „daß mit dem $\S 43$ a die Rechtsgrundlage für die Phase der Diagnostik und den Behandlungsplan geschaffen wird und daß sich das, was an Therapie gegeben wird, nach dem Leistungsrecht der gesetzlichen Krankenkassen richtet"“, Verh. d. BT, 12. WP, 52. Sitzung, 30.10.1991, S. 4273 1.Sp.
} 
Arzt angeordnet und von ihm zu verantworten ist“. Wegen ,normative[r] Untauglichkeit", so Schulin, könne § 43a SGB V nicht diese allgemeinen Regeln des Leistungsrechts verdrän$\operatorname{gen}^{27}$.

Die Entstehungsgeschichte der Novelle von 1991 spricht jedoch eher gegen die Position von Schulin. Der Bundesrat hatte u.a. wegen der bereits vom Bundestag beschlossenen Regelung des $\S 43$ a Einspruch gegen das Änderungsgesetz erhoben und vorgeschlagen, den neuen $\S 43 \mathrm{a}$ ebenso umfassend abzufassen wie den vorherigen $\S 119$ Abs. 2 (a.F.) ${ }^{28}$. Dieser Vorschlag war vom Vermittlungsausschuss jedoch mit dem Argument nicht aufgegriffen worden, die „vom Bundesrat begehrte Ausweitung der sozialpädiatrischen Leistungen hätte lediglich zu einer Verlagerung der Kosten vom Sozialhilfeträger auf die GKV geführt, ohne daß damit eine Leistungsverbesserung für die betroffenen Kinder verbunden gewesen wäre" ${ }^{\text {"29 }}$. Man hat also damals bewusst eine eingeschränkte Leistungspflicht der Krankenkassen nur für die „Diagnosephase ${ }^{\text {"30 }}$ ins Gesetz geschrieben.

\section{Plausibilität des „Umfassungsgrundsatzes“ seit 2009}

Die Einführung des $§ 43 a$ Abs. 2 SGB V im Jahr 2009 legt nun aber m.E. eine Änderung der Auslegung des Gesetzes im Sinne des „Umfassungsgrundsatzes“ nahe, also im Sinne einer Leistungs- und Finanzierungsverantwortung der Krankenkassen für nichtärztliche sozialpädiatrische Leistungen in Sozialpädiatrischen Zentren auch jenseits von Diagnostik und Behandlungsplanung, wenn diese Leistungen unter ärztlicher Verantwortung erbracht werden.

Im Blick hatte der Gesetzgeber bei der Einführung der Vorschrift zwar allein das Versorgungssegment der auf der Grundlage der Sozialpsychiatrie-Vereinbarung tätigen Vertragsärzte. Die bei niedergelassenen Fachärzten für Kinder- und Jugendpsychiatrie entstandenen und von den Krankenkassen insgesamt über die Gesamtvergütung finanzierten multiprofessionellen Teamstrukturen sollten abgesichert werden. Eine Mischfinanzierung der Praxen niedergelassener Ärzte unter Einbeziehung von Sozialhilfemitteln wurde nicht einmal im Ansatz in Erwägung gezogen.

Wenn aber die Krankenkassen Maßnahmen interdisziplinärer Behandlungsteams unter Einschluss von Heilpädagogen, Sozialarbeitern und Sozialpädagogen insgesamt verantworten und finanzieren, sofern ein solches Team in der Praxis eines niedergelassenen Facharztes für Kinder- und Jugendpsychiatrie tätig wird, ist es schwer einzusehen, dass ein in gleicher Weise zusammengesetztes Team von den Kassen nur zu 85 \% verantwortet und finanziert werden sollte, wenn das Team in einem sozialpädiatrischen Zentrum arbeitet. Deshalb spricht viel dafür, das Gesetz im Sinne einer vollen Leistungsverantwortung der Krankenkassen auszulegen. Der Wortlaut des $\S 43 a$ Abs. 2 SGB V lässt diese Auslegung insofern ohne Weiteres zu, als es sich sowohl bei Maßnahmen eines Teams des niedergelassenen Facharztes für Kinder- und Jugendpsychiatrie wie auch bei Maßnahmen eines Teams im Sozialpädiatrischen Zentrum um ambulante Behandlung unter ärztlicher Verantwortung handelt.

\footnotetext{
${ }^{27}$ Schulin, aaO (Fn. Xxx), S. 123, Nr. 5.

${ }^{28}$ BT-Drs. 12/1526 (Unterrichtung des Bundestages durch den Bundesrat über die Anrufung des Vermittlungsausschusses u.a. wegen dieser Frage).

${ }^{29}$ So der Abgeordnete Menzel (FDP) am 27.11.1991 im Bundestag, Verh. d. BT, 12. WP, 60. Sitzung, S. 5087, r.Sp.

${ }^{30}$ So die Begrifflichkeit des Abgeordneten Jagoda am 30.10.1991 im Bundestag, Verh. d. BT, 12. WP, 52. Sitzung, S. 4273, 1.Sp.; zu Jagodas abweichender Auffassung betreffend die Durchführungsphase bereits oben Fn. Xxx.
} 
Allerdings muss diese Auslegung mit der Schwierigkeit umgehen, dass sich $\S 43 \mathrm{a}$ Abs. 2 SGB V dem Wortlaut nach lediglich auf psychiatrische Behandlung bezieht. Man ist versucht, daraus den Schluss zu ziehen, es sei zwischen sozialpädiatrisch-psychiatrischer und sonstiger sozialpädiatrischer Versorgung zu unterscheiden, und nur im erstgenannten Fall müssten auch die Leistungen der nicht-ärztlichen Teammitglieder umfassend von den Krankenkassen finanziert werden, im zweiten Fall hingegen - Sozialpädiatrie ohne psychiatrischen Bezug - seien die Krankenkassen nur für die Finanzierung der Diagnosephase zuständig. Demgegenüber erscheint eine Deutung plausibler, die die Situation der kranken oder von Krankheit bedrohten Kinder zum Ausgangspunkt nimmt: wenn die Schwere der Krankheit es nahelegt, dass therapeutische Maßnahmen in multiprofessionellen Teams unter ärztlicher Verantwortung durchgeführt werden, dann sollen alle Elemente der Therapie, ärztliche wie nichtärztliche, in die Leistungs- und Finanzierungsverantwortung der Krankenkassen fallen, und zwar unabhängig davon, ob das Team in der Praxis eines Facharztes für Kinder- und Jugendpsychiatrie tätig wird oder aber in einem Sozialpädiatrischen Zentrum. Dies wäre ein „objektiv-teleologischer“ Deutungsansatz, der an $§ 119$ Abs. 2 SGB V anknüpft, wo die Behandlung der in Bezug auf Art, Schwere oder Dauer komplizierten Erkrankungen den Sozialpädiatrischen Zentren zugewiesen wird.

\section{Entscheidung des LSG Berlin-Brandenburg im Sinne des „Umfassungsgrundsatzes“}

Das LSG Berlin-Brandenburg hat den streitbefangenen Schiedsspruch als rechtmäßig betrachtet und damit die von der Schiedsstelle vorgenommene Auslegung des Gesetzes im Sinne des „Umfassungsgrundsatzes“ gebilligt. Dieses Ergebnis wird allerdings im Urteil nur äußerst knapp begründet ${ }^{31}$ : Zunächst wird eine Klausel aus der Vereinbarung der Parteien für 2014 zitiert, nach der ,die Leistungen des SPZ [...] die ärztlich verantwortete Diagnostik, Behandlungsplanung und Therapie [umfassen] sowie die auf ärztliche Veranlassung erbrachten nichtärztlichen sozialpädiatrischen Leistungen, soweit sie gemäß § 119 SGB V i.V.m. § 43a SGB $\mathrm{V}$ in den Leistungsrahmen der gesetzlichen Krankenversicherung fallen“. Später heißt es dann: „Nicht ersichtlich ist aber auch, dass die streitgegenständliche Vergütungsvereinbarung überhaupt Leistungspositionen umfasst, auf die die versicherten Kinder und Jugendlichen nicht nach § 43a Abs. 1, Abs. 2 SGB V einen Anspruch hätten“. Von einer klaren Bestimmung des Auslegungsproblems und von einer Entfaltung inhaltlicher Argumente zur Lösung des Problems sieht das LSG ab. Gleichwohl: wie oben ausgeführt, erscheint das Gesetzesverständnis des Gerichts im Ergebnis durchaus plausibel - vor allem angesichts des $\S 43 \mathrm{a}$ Abs. 2 SGB V, den das LSG zumindest erwähnt.

Wenn auch das LSG keine inhaltliche Begründung für sein Gesetzesverständnis gibt, so liefert es doch immerhin ein autoritatives Argument, denn es bezieht sich auf Rechtsprechung des BSG, und zwar auf eine Formulierung aus einem Urteil des 6. Senats des BSG aus dem Jahr 2011, die wie folgt lautet: „, Für die Vergütung der sozialpädiatrischen Leistungen, die von Vertragsärzten und Frühförderstellen verantwortet werden, gilt § 85 Abs. 2 S. 4 SGB V, während für die ärztlichen und nicht-ärztlichen sozialpädiatrischen Leistungen der SPZ bei Diagnostik, Beratung, Förderung und Therapie die Regelungen des $\S 120$ Abs. 2 ff SGB V maßgebend sind“332. Mit Recht ordnet das LSG diese vierfache Aufzählung zum vergütungsrelevanten Leistungsumfang als ein obiter dictum ein, denn im Fall des BSG war nicht die Vergütung für Leistungen eines Sozialpädiatrischen Zentrums Streitgegenstand; vielmehr hatte sich ein

\footnotetext{
${ }^{31} \mathrm{Vgl}$. in diesem Heft S. $\mathrm{xx}$

${ }^{32}$ BSG, Urt. v. 29.6.2011, B 6 KA 34/10 R, SozR 4 - 2500 § 119 Nr. 1, Rn 10.
} 
Krankenhaus gegen die auf fehlenden Bedarf gestützte Ablehnung einer von ihm beantragten Ermächtigung gewehrt. Eine Entscheidung des BSG, die sich in entscheidungserheblicher Weise mit dem Umfang der Vergütungspflicht der Krankenkassen befasst, gibt es bisher nicht $^{33}$.

Die zitierte Stelle aus dem Urteil des 6. Senats kann im Sinne der Auffassung des LSG verstanden werden. Sie ist allerdings recht knapp ausgefallen und ermangelt auch einer Begründung. Es ist deshalb verwunderlich, dass das LSG die Revision gegen sein Urteil nicht zugelassen hat, geht es doch um eine grundsätzlich bedeutsame Rechtsfrage, die Klärung verdient hätte. Ein bloßes obiter dictum des BSG lässt den Klärungsbedarf jedenfalls nicht entfallen ${ }^{34}$. Die unterlegene Krankenkasse hatte allerdings wohl kein Interesse an einer genaueren Klärung, denn das Urteil des LSG ist rechtskräftig geworden ${ }^{35}$, offenbar ohne dass eine Nichtzulassungsbeschwerde erhoben worden wäre. In einem Parallelverfahren hatte die Kassenseite von vornherein von einer gerichtlichen Klärung abgesehen ${ }^{36}$.

\section{Zur Abrundung: Komplikationen durch das „Frühförderungsrecht“"}

Die hier gutgeheißene Lösung des LSG kann zu Schwierigkeiten führen, wenn ein Sozialpädiatrisches Zentrum als Frühförderstelle im Sinne des $\S 4$ FrühV behinderten oder von Behinderung bedrohten Kindern im Vorschulalter Komplexleistungen erbringt (vgl. $\S \S 6$ und 8 FrühV sowie $\S \S 46$ Abs. 3 und 79 SGB IX n.F.). Hinsichtlich der nicht-ärztlich zu erbringenden Elemente solcher Leistungen sind Absprachen über eine Kostenteilung gesetzlich vorgesehen ( 46 Abs. 5 S. 3 SGB IX n.F.), und zwar seit dem 1.1.2018 ausdrücklich auch in Bezug auf heilpädagogische Leistungen der Sozialpädiatrischen Zentren, indem nämlich der Entgeltanteil für diesen Teil der Komplexleistung der Zentren auf höchstens $20 \%$ begrenzt wird ${ }^{37}$. Das bedeutet offenbar, dass die Krankenkasse mit $80 \%$ der Kosten für die Komplexleistung belastet werden sollen, nicht jedoch mit den Kosten für den heilpädagogischen Leistungsanteil (20\%).

Das im SGB IX angelegte Kostenteilungsmodell passt offenkundig nicht zu dem von Schiedsstelle und LSG zu Grunde gelegten „Umfassungsgrundsatz“. Im entschiedenen Fall wurde das Problem auf der Ebene der Sachverhaltsfeststellungen gelöst: Das LSG hat die Darstellung des Zentrums akzeptiert, dass es keine Komplexleistungen als Frühförderstelle erbringe.

Schwieriger ist die Lage, wenn ein Sozialpädiatrisches Zentrum auch als Frühförderstelle tätig wird und als solche auch Komplexleistungen einschließlich heilpädagogischer Leistungselemente erbringt, für die nach dem gesetzlichen Modell - beredtes Schweigen des § 79 SGB IX n.F. - die Notwendigkeit ärztlicher Verantwortung gerade nicht erforderlich ist. Bei einer solchen Gestaltungsform liegt es nicht nahe, eine Verantwortung der Krankenkassen für nichtmedizinische Leistungsanteile anzunehmen. Die Frage, ob in einem Sozialpädiatrischen Zent-

\footnotetext{
${ }^{33}$ Mit der Kalkulation der Vergütung der Leistungen der Sozialpädiatrischen Zentren befasst sich auch das Urteil des BSG v. 13.5.2015, B 6 KA 20/14 R, E 119, S. 43 ff., das die aus dem Recht der sozialen Pflegeversicherung bekannte Figur des ,externen Vergleichs“ in das Vergütungsrecht der Sozialpädiatrischen Zentren übertragen hat; die Frage, welche Leistungselemente im Einzelnen in die Kalkulation einbezogen werden können, wird in diesem Urteil jedoch nicht behandelt.

${ }^{34}$ Krasney/Udsching/Groth, Handbuch des sozialgerichtlichen Verfahrens, 7.Aufl., 2016, S. 348, Rn. 65.

${ }^{35}$ Auskunft der Pressestelle des LSG.

${ }^{36}$ Der Schiedsspruch der Schiedsstelle nach § 18a KHG für das Land Brandenburg v. 9.5.2017 - 01/2017 (2015 und 2016), KrV 2017, S. 212 ff., war, wie es dort (S. 212, r. Sp.) heißt, von keiner der Parteien angefochten worden.

${ }^{37}$ Zuvor fand sich diese Regelung in $\S 9$ Abs. 3 FrühV a.F.; vgl. dazu BT-Drs. 18/9522, S. 252.
} 
rum, für das die ständige ärztliche Leitung konstitutiv ist (so § 119 Abs. 1 SGB V), der Aufbau einer gewissermaßen arztfreien Abteilung wirklich sinnvoll ist, erscheint zweifelhaft, mag aber hier letztlich offenbleiben.

\section{Ergebnis}

Nach allem bleibt festzuhalten, dass das Urteil des LSG mit Recht dem Sozialpädiatrischen Zentrum gestattet, auch die Kostenanteile für nicht-ärztliche sozialpädiatrische Leistungen, insbesondere auch für von Heil- oder Sozialpädagogen durchgeführte therapeutische Maßnahmen, in die von den Krankenkassen zu tragenden Vergütungspauschalen einzukalkulieren. Für dieses vom LSG nicht inhaltlich, sondern nur mit Hinweis auf ein obiter dictum des BSG begründete Ergebnis lassen sich auch inhaltliche Argumente finden, die zwar ein wenig umständlich sind, aber doch vertretbar erscheinen. Eine deutlichere Fassung der gesetzlichen Regelungen, insbesondere des $\S 43 \mathrm{a}$ SGB V, im Sinne einer eindeutigen leistungsrechtlichen Verankerung des „Umfassungsgrundsatzes“ wäre im Interesse der Rechtssicherheit allemal wünschenswert und würde die Praxis der Vergütungsverhandlungen gewiss erleichtern.

(Dieses Manuskript ist erschienen in der Zeitschrift Die Sozialgerichtsbarkeit, Heft 7 2019, S. $410-415)$. 